\title{
Fraktionierte $\mathrm{CO}_{2}$-Laserbehandlung der Haut Fractional $\mathrm{CO}_{2}$-Laser Treatment for Skin Resurfacing
}

\begin{abstract}
Autoren
S. Steckmeier ${ }^{1}$, G. G. Gauglitz ${ }^{1}$, J. Poetschke ${ }^{2}$

Institute

1 Klinik und Poliklinik für Dermatologie und Allergologie, Ludwig-Maximilians-Universität München

2 Plastische und Handchirurgie mit Schwerbrandverletzungszentrum, Klinikum St. Georg, Leipzig

Verfahren eine effektive Neokollagenese bei minimalen Ausfallzeiten und guter Verträglichkeit für die Patienten zu erreichen. Der besonders bei den fraktionierten $\mathrm{CO}_{2}$-Lasern ausgeprägte „Shrinking“-Effekt führt zu einer signifikanten Stimulation des Kollagenumbaus und einer Straffung der Haut. Bis heute zählt er zu den am besten untersuchten und effektivsten Behandlungsverfahren im Anti-Aging-Bereich und in der Therapie von atrophen und hypertrophen (Brand-)Narben.
\end{abstract}

\author{
Bibliografie \\ DOI https://doi.org/10.1055/s-0043-112537 | \\ Akt Dermatol 2017; 43: 418-426 \\ (c) Georg Thieme Verlag KG Stuttgart · New York \\ ISSN 0340-2541
}

Korrespondenzadresse

Dr. med. Stephanie Steckmeier, Klinik und Poliklinik für Dermatologie und Allergologie, Klinikum der Universität München, Ludwig-Maximillians-Universität,

Frauenlobstraße 9-11, 80337 München

stephanie.steckmeier@med.uni-muenchen.de

\section{ZUSAMMENFASSUNG}

Zur Verbesserung der Hautstruktur und zur allgemeinen Glättung bzw. Straffung der Haut (vor allem der Gesichtshaut) kommen heutzutage zunehmend fraktionierte Laserverfahren zum Einsatz. Durch das Prinzip der fraktionierten Photothermolyse ist im Vergleich zu den klassisch ablativen

\section{ABSTRACT}

To improve the skin structure and to achieve overall smoothening or toning of the skin, fractional lasers are frequently utilized. Due to the technique of fractional photothermolysis, an effective neocollagenesis is achieved with minimal downtime and good tolerability for the patients compared to the conventional, fully ablative methods. Depending on the employed settings, fractional $\mathrm{CO}_{2}$-laser treatments will initiate a significant stimulation of collagen remodeling and will ultimately result in skin tightening. To date, this technology represents one of the best-studied and most effective treatment procedures in the anti-aging field and has become one of the central options for the treatment of atrophic - and hypertrophic scars.

\section{Einleitung}

Laser werden in der Dermatologie seit nunmehr 50 Jahren verwendet. Aufgrund ihrer unterschiedlichen Wellenlängen und somit selektiven Ausrichtung auf die verschiedenen Haut-Chromophoren sind sie zu einer bevorzugten Therapieoption für viele Hautveränderungen geworden, seien sie vaskulärer, pigmentärer, entzündlicher, prä-maligner oder kosmetischer Natur. Die Technologie und das Design der verschiedenen Lasergeräte haben sich in den letzten Jahren rasant weiterentwickelt. Trotz aller Innovationen bleibt ein solides Grundverständnis der jeweiligen Wellenlänge und der verwendeten Technologie von entscheidender Bedeutung, um die gewünschten Effekte am Patienten nebenwirkungsarm zu erreichen. Der im Folgenden beschriebene Kohlendioxid-Laser $\left(\mathrm{CO}_{2}\right.$-Laser $)$ hat insbesondere seit der Einführung der fraktionierten Photothermolyse in den letzten Jahren aufgrund seiner populären Indikationen erneut an Bedeutung gewonnen.

\section{Historie}

Der dem Laserprinzip zugrunde liegende Effekt der stimulierten Emission wurde erstmals 1917 von Einstein beschrieben. Weitere Grundlagenforschung führte zur Entwicklung des ersten Rubinlasers durch Maiman 1960. Leon Goldman gilt als der Pionier für die Einführung der Laser in die Dermatologie und bereits 1967 erschien sein klassisches Buch: „Biomedical Aspects of the Laser“. Im selben Jahr wurde der erste Gaslaser von Javan, Bennet und Herriott geschaffen. Schon kurze Zeit nach der Entwicklung des ersten Lasers wurde sein Nutzen für die Medizin entdeckt. Ein weiterer Meilenstein war die Publikation des Prinzips der selektiven Photothermolyse durch Anderson und Parrish 1983 [1]. Das erstmals 2004 von Manstein et al. beschriebene Prinzip der fraktionierten Photothermolyse hat die Anwendungsmöglichkeiten für die klassisch ablativen Laserverfahren wie den $\mathrm{CO}_{2}$-Laser oder den Erbium-doped yttrium aluminium garnet Laser (Er:YAG-Laser) revolutioniert. 


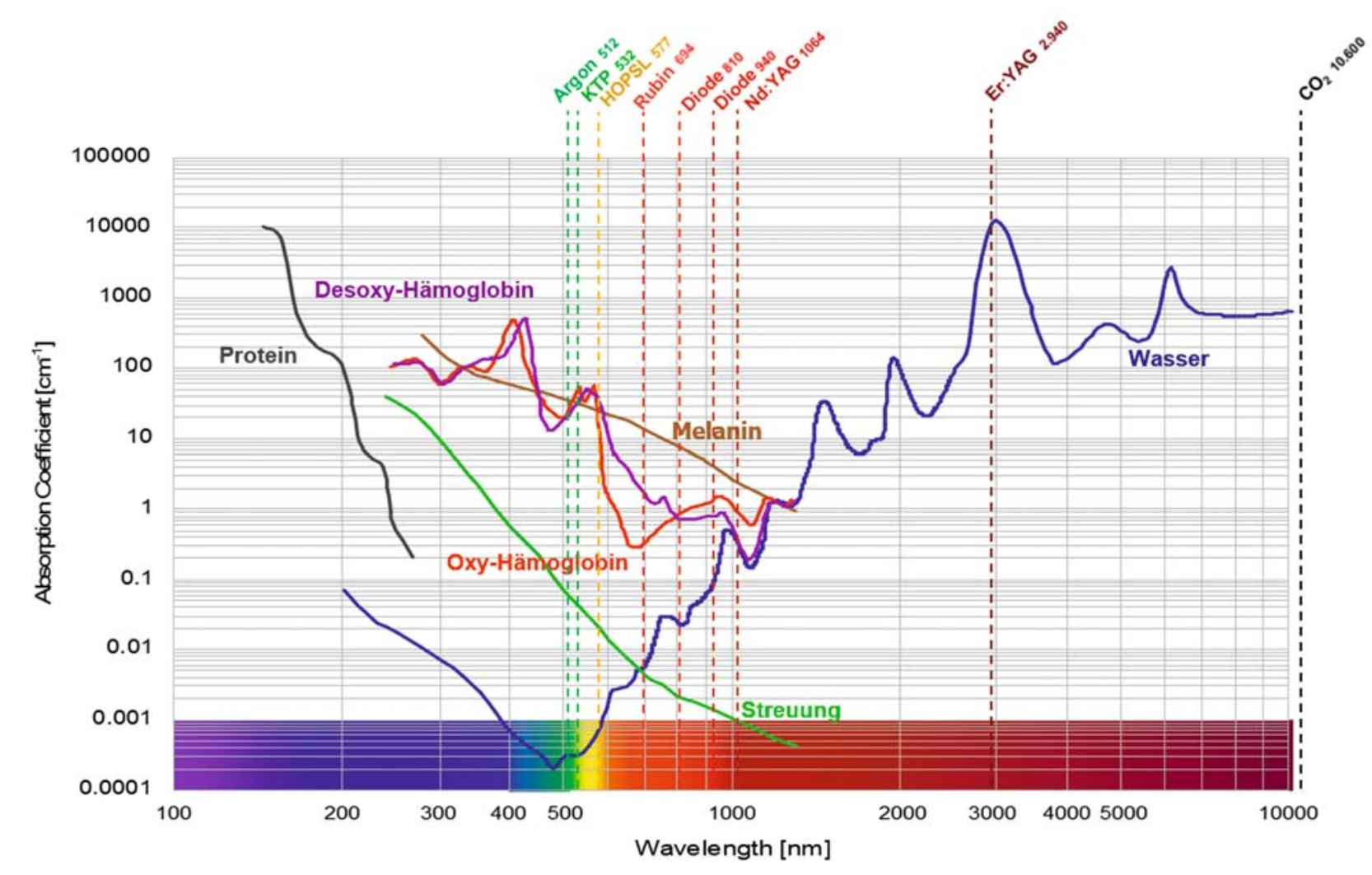

- Abb.1 Prinzip der selektiven Photothermolyse (mit freundlicher Genehmigung von Asclepion Laser Technologies, Jena).

\section{Methodik/physikalische Grundlagen}

Der Terminus Laser ist ein Akronym für light amplifikation by stimulated emission of radiation. Übersetzt: Durch stimulierte (angeregte) Emission von Strahlung findet eine Lichtverstärkung statt. Beim Durchlauf eines Photons durch verschiedene Substanzen wird die Emission eines weiteren Photons derselben Wellenlänge stimuliert. Diese Substanzen bilden das aktive Medium eines Lasers, das ein Festkörper (z.B. Rubinkristall), eine Flüssigkeit (z. B. Farbstofflösung Rhodamin 6G) oder ein Gas ( $\mathrm{CO}_{2}$, Argon, Krypton) sein kann. Beim Gas-Laser wird das Gasgemisch (z. B. $\mathrm{CO}_{2}$-Stickstoff-Helium) durch eine externe Stromquelle oder zum Beispiel Radiofrequenz zum Schwingen angeregt [1]. Dadurch kommt es zur weiteren Emission von Photonen, die zwischen den Spiegeln im Inneren des Resonators reflektiert werden, sich verstärken und letztendlich zur Erzeugung eines Laserstrahls gebündelt werden. Das dadurch erzeugte Laserlicht ist anders als natürliches Licht oder das einer Glühbirne etwa sehr intensiv, einfarbig (monochromatisch) und kohärent [2]. Die Effekte der Laserstrahlung im Gewebe hängen ab von der Laserleistung $\mathrm{P}$ am Behandlungsort, der Strahlfläche A und der Bestrahlungszeit t. Daraus ergibt sich dann die Leistungsdichte oder Intensität: I=P/A (W/ $\left.\mathrm{cm}^{2}\right)$ und die Energiedichte oder Bestrahlungsdosis $D=1 \times t\left(J / \mathrm{cm}^{2}\right)$ [1]. Voraussetzung für den therapeutischen Einsatz ist die
Absorption der Laserstrahlung einer bestimmten Wellenlänge in der Zielstruktur ( $>$ Abb. 1).

Die wesentlichen Absorber in der Haut sind Hämoglobin $\left(\mathrm{HbO}_{2}\right.$ und Met- $\left.\mathrm{Hb}\right)$, Melanin, Wasser, exogene Farbstoffe (Tätowierungsfarben, Medikamente) oder Photosensibilisatoren. Je nach Wahl der physikalischen Parameter resultieren im Gewebe photochemische Prozesse, thermisch-destruktive Effekte wie Koagulation, Disruption, Vaporisation und Ablation oder selektive Photothermolyse. Während der Laserbestrahlung kommt es durch die Absorption der Lichtenergie neben einer Erhitzung der Zielstruktur in Abhängigkeit von der Behandlungsdauer auch zur Wärmeleitung in die unmittelbare Umgebung. Temperaturen zwischen $60-90^{\circ} \mathrm{C}$ führen zur Koagulation von Gewebe mit Denaturierung von Proteinen und Kollagen. Bei der Vaporisation führen Temperaturen von über $100^{\circ} \mathrm{C}$ zur Verdampfung von Gewebe. Bei weiterer Temperaturerhöhung kommt es zu einem Gewebedefekt mit einer oberflächlichen Karbonisationsschicht, unter der eine unterschiedlich breite Koagulationszone liegt. Liegt die Bestrahlungszeit unterhalb der thermischen Relaxationszeit (= die Zeit, die die Struktur benötigt, um die durch den Laserstrahl deponierte Energie wieder durch Wärmeleitung in die Umgebung abzugeben), kann in dieser Zeit keine Wärme an die Umgebung abgegeben werden, die gesamte eingestrahlte Energie wird auf die Zielstruktur beschränkt und kann diese selektiv zerstören [1]. Eine Chromophore oder eine be- 
stimmte Zielstruktur kann somit selektiv, mit minimaler thermischer Gewebeschädigung im Randbereich, zerstört werden, wenn die Wellenlänge des Laserlichts optimal an das Absorptionsmaximum der Zielstruktur angepasst ist, die Laserexpositionszeit (Pulslänge) unterhalb der thermischen Relaxationszeit der Zielstruktur liegt und eine ausreichende Energiedosis gegeben ist. Dieses Konzept der selektiven Photothermolyse legte den Grundstein für ein neues Verständnis zur Interaktion zwischen Laserstrahl und Gewebe und revolutionierte in den 80er-Jahren die Lasertechnologie.

\section{Funktionsweise des $\mathrm{CO}_{2}$-Lasers, vom konventionellen ablativen Verfahren zur fraktionierten Photothermolyse}

Klassisch ablative Verfahren zur Laserbehandlung von Falten, Aknenarben und anderen Hautveränderungen gutartiger (z. B. Alterswarzen, dermale Muttermale, Xanthelasmen) wie prämaligner (z.B. aktinische Keratosen, M. Bowen) Natur haben eine jahrzehntelange Geschichte. Hierfür werden Er:YAG-Laser (Infrarotlicht der Wellenlänge $2940 \mathrm{~nm}$ ) und insbesondere $\mathrm{CO}_{2}$-Laser (Infrarotlicht der Wellenlänge $10640 \mathrm{~nm}$ ) verwendet, die eine hohe Absorptionsrate für Wasser aufweisen. Die Absorption der Laserstrahlen führt in den stark wasserhaltigen Hautzellen zu deren explosionsartiger Verdampfung (Vaporisation) und somit zur Abtragung der behandelten Hautschichten. Der Prozess der kontrollierten Abtragung epidermaler Strukturen bis in die Dermis wird als Laser-Skin-Resurfacing bezeichnet. Die Hauterneuerung erfolgt über die Reepithelialisierung durch Keratinozyten, welche von den Hautanhangsgebilden (Haarfollikeln) ausgeht. Obwohl beide Laser ihr Absorptionsmaximum im Wasser haben, der sich seit 1996 auf dem Markt befindende Er:YAG-Laser sogar um das 10-16-Fache stärker als der $\mathrm{CO}_{2}$-Laser [2], hat der Er:YAG-Laser praktisch nur eine Vaporisationswirkung, jedoch effektiv nur eine geringe Hitzewirkung auf das umliegende Gewebe. Dies ist auch als Prinzip der „kalten Abtragung“ bekannt. Da die thermische Restnekrose nur 20-50 Mikrometer beträgt und kleine Blutgefäße somit nicht verschlossen werden, kommt es beim Er:YAG-Laser rasch zu Nachblutungen [1]. Der $\mathrm{CO}_{2}$-Laser hingegen führt durch seine ausgesprochen thermische Wirkung zu Entzündungsprozessen in tieferen Hautschichten (Dermis) und somit zu einer Stimulation von Fibroblasten, was die Neubildung von Kollagen und Elastin anregt. Die Hitzekoagulation an der Basis des eigentlichen Abtragungsdefekts führt zu einem sogenannten „Shrinking“-Effekt, worunter man eine Neustrukturierung und Straffung des Bindegewebes durch die Reparatur und Reorganisation hitzegeschädigter Matrixproteine versteht. Diese Effekte machen den $\mathrm{CO}_{2}$-Laser besonders effektiv, wenn es darum geht, faltige Haut zu glätten oder Narben zum Schrumpfen zu bringen [3]. Darüber hinaus treten bei der Behandlung mit $\mathrm{CO}_{2}$-Lasern keine Blutungen auf, da geplatzte Äderchen durch seine Wärmeentwicklung sofort verödet werden (Blutstillung).

Die ersten Laser arbeiteten im Dauerstrich (continuous wave) cw)-Modus, der einen kontinuierlichen Laserstrahl erzeugt, welcher insbesondere von dermalen Zielstrukturen absorbiert wird. Der limitierende Faktor ist jedoch, dass die Laserenergie durch Wärmeleitung auch auf benachbarte Hautstrukturen einwirkt. Die 1964 entwickelten cw- $\mathrm{CO}_{2}$-Laser $(10600 \mathrm{~nm})$, bei denen die Laserstrahlung über Spiegelarme um ein Fokussierhandstück auf die Haut gebracht wird, erreichen im Fokus einen extrem kleinen Strahldurchmesser, der als Lichtskalpell zum blutungsfreien Schneiden verwendet werden kann [2]. Im defokussierten Modus wurde die Haut für das erste Laser-Skin-Resurfacing abgetragen. An der Basis des Abtragungsdefekts kommt es zu der oben beschriebenen thermischen Restnekrose unterhalb der eigentlichen Ablationszone von bis zu 0,3 mm [1]. Dieser zunächst vorteilhafte „Kollateralschaden“ im angrenzenden Gewebe ist jedoch nicht nur positiv zu betrachten. Durch die Hitzeentwicklung ist die Behandlung für den Patienten äußerst schmerzhaft. Es kann zur Narbenentstehung kommen sowie, besonders in empfindlichen Arealen (z. B. periorbital), zu kosmetisch unschönen Demarkierungslinien zwischen behandelter und unbehandelter Haut. Aus diesen Gründen und durch die Entdeckung des Prinzips der selektiven Photothermolyse erst ermöglicht, wurde die Lasertechnologie zu den heute hauptsächlich genutzten gepulsten $\left(\mathrm{CO}_{2}\right.$-) Lasern weiterentwickelt. Anstelle eines kontinuierlichen Laserstrahls wird hier eine sehr schnelle Folge kurzer Impulse mit hoher Energiedichte emittiert. Die heutigen Kurzpulstechnologien (supergepulste und ultragepulste) ermöglichen eine selektive, nebenwirkungsarme, tiefe Vaporisation (Eindringtiefe bis $4 \mathrm{~mm}$ beim UltraPulse ${ }^{\circledR}$, Lumenis), d. h. maximale spezifische Wirkung auf das Zielgewebe mit minimaler Hitzeschädigung im umliegenden Gewebe, was der Patientensicherheit zugute kommt und Beschwerden während und infolge der Laserbehandlung reduziert. So ein Prozess ist nur dann möglich, wenn die Pulsenergie gleichmäßig hoch und die Einwirkzeit des Laserstrahls sehr kurz ist, genauer gesagt unter der thermischen Relaxationszeit liegt, wie es bei den ultragepulsten $\mathrm{CO}_{2}$-Lasern der Fall ist.

Die fachgerechte Behandlung mit diesen Lasern führt im Allgemeinen zu guten Ergebnissen. Die konventionelle, ablative Laser-Technologie bringt aber, wie schon erwähnt, einige Einschränkungen und Komplikationen mit sich. Ähnlich wie bei den anderen invasiven Therapieoptionen, der chirurgischen Dermabrasion und den (tiefen) chemischen Peelings, bei denen ebenfalls die gesamte Hautfläche abgetragen wird, benötigt die Abheilung zum Teil mehrere Wochen, in denen der Patient mit Schwellungen und Rötungen des Gesichts rechnen muss und zum Teil wochenlang seinen sozialen Verpflichtungen nur eingeschränkt nachgehen kann. Diese sogenannte „downtime“ ist in der heutigen, schnelllebigen Gesellschaft kaum noch tolerierbar. Zudem ist die Gefahr von Narbenbildung, lokalen Infektionen und bleibenden Pigmentverschiebungen sowie der schon erwähnten kosmetisch unschönen Demarkierungszonen bei vollflächig wirkenden Therapieverfahren erhöht.

Aus dem Wunsch nach sicheren, nebenwirkungsärmeren aber zugleich effektiven Behandlungsmethoden entwickelte sich die fraktionierte Photothermolyse [4, 5].

Mittels eines speziellen Laserkopfes erfolgt die Applikation des Laserlichts nicht wie bisher flächig, sondern durch einen in viele tausend einzelne Lichtsäulen aufgeteilten (fraktionierten) Laserstrahl. Die hierbei entstehenden zahlreichen Mikro- 

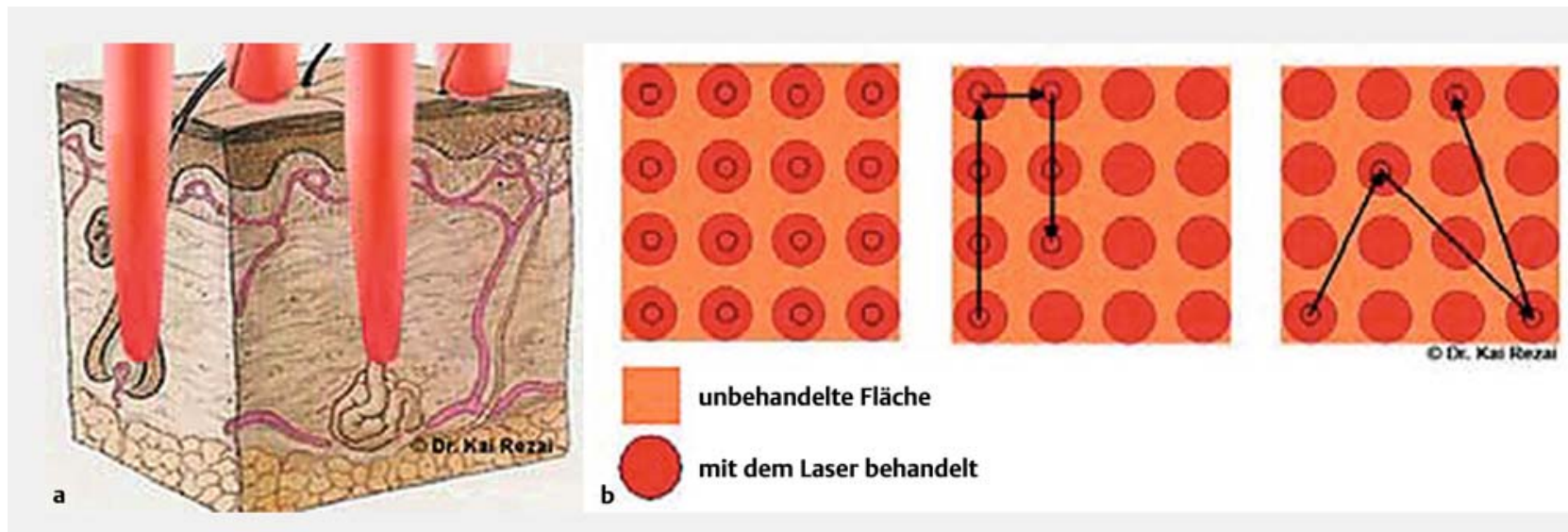

unbehandelte Fläche

b

mit dem Laser behandelt

- Abb. 2 a Fraktionierte Photothermolyse: Schema der Behandlungsmethode. b Die verschiedenen Verfahren schematisch nebeneinander dargestellt (Quelle: Rezai K. Fraktionierte $\mathrm{CO}_{2}$-Lasertherapie bei lichtgeschädigter Haut und Falten. Fachbeitrag Lasertherapie, Face 2009; 4: 46).

wunden werden als mikrothermale Behandlungszonen (MTZ= microthermal treatment zones) bezeichnet ( $\boldsymbol{A} \mathbf{A b b} \mathbf{2} \mathbf{a})$.

Die MTZ sind immer von gesundem, durch das Laserlicht nicht beschädigtem Gewebe umgeben, von dem aus sich das zerstörte Gewebe wieder regenerieren kann. Die vielen kleinen Nekrosezonen stimulieren im Zuge der Wundheilung die Bildung von neuen Kollagenfasern, werden zu Mikronarben, schrumpfen und führen so zu einer Straffung der Haut. Zugleich werden die behandelten Zellen abgestoßen. Durch die unversehrt gebliebenen Hautbrücken entsteht weniger Wundschorf als bei den klassisch, vollflächig wirkenden Lasertechnologien. Dies bewirkt zum einen eine schnellere Heilung, weniger Nebenwirkungen, deutlich kürzere Ausfallzeiten (je nach verwendeter Energie und Dichte 5-10 Tage) und geringere Schmerzen [6]. Hier werden also die Vorteile des $\mathrm{CO}_{2}$-LaserSkin-Resurfacings mit den Vorteilen der fraktionierten Technik kombiniert.

Die molekularen Vorgänge nach der Behandlung sind vielseitig und bewirken eine Veränderung verschiedener Zytokinund Wachstumsfaktorkonzentrationen (Heat Shock Proteine, Matrixmetalloproteinasen und Transforming Growth Factor [TGF] beta 3), eine Wiederherstellung der ursprünglichen Kollagenarchitektur und eine Zunahme von elastischen Fasern [7, 8]. Bezüglich der aktuell auf dem Markt verfügbaren $\mathrm{CO}_{2}$-Laser gibt es jedoch zwischen den unterschiedlichen Fabrikaten erhebliche Unterschiede. Während manche Systeme gleich einem Stempel alle MTZ im selben Augenblick applizieren, sind andere Geräte in der Lage, bahnenweise zu arbeiten oder das vorgegebene Scannermuster randomisiert (willkürlich) zu applizieren ( $\triangleright$ Abb. $\mathbf{2} \mathbf{b}$ ).

Letzterer Modus bietet letztlich die größten Vorteile, da die Applikation der einzelnen MTZ zeitlich am weitesten auseinander liegt, was die thermische Erwärmung der einzelnen unbehandelten Gewebebrücken deutlich reduziert.

Die Suche nach noch schonenderen Verfahren, aufgrund der für viele Patienten immer noch signifikanten Downtime der ablativen, fraktionierten Systeme, führte zur Entwicklung von nicht-ablativen, fraktionierten Verfahren. Inzwischen stehen eine Vielzahl unterschiedlicher Wellenlängen zur Verfügung: 1,064-, 1,320- und 1,440-nm Nd:YAG-Laser oder 1,410-, 1,550-, 1565-nm Er-glass Laser [9].

Die nicht-ablative fraktionierte Lasertherapie nutzt die Induktion des epidermalen wie dermalen Remodelings durch umschriebene dermale Erhitzung bei epidermaler Kühlung [9]. Bei diesen Verfahren bleibt die Epidermis intakt - somit verringert sich die Downtime und das Risiko von Nebenwirkungen, gleichzeitig aber auch deren Effektivität. Indikationen stellen feine Fältchen, Pigmentunregelmäßigkeiten und der Wunsch nach allgemein leichter Straffung der Haut dar. Die richtige Patientenselektion ist entscheidend für die Auswahl des geeigneten Laserverfahrens und somit für die Patientenzufriedenheit.

Bei tieferen Falten, z.B. der Perioral-/Periorbitalregion, bei ausgeprägter Elastose sowie bei atrophen Aknenarben gehört der Einsatz von ablativen fraktionierten $\mathrm{CO}_{2}$-Lasern hinsichtlich ihres Nutzen/Risiko-Profils inzwischen zu den am besten untersuchten und effektivsten Behandlungsverfahren [2,6].

\section{Praktische Durchführung der $\mathrm{CO}_{2}$-Laser- Behandlung, richtige Patientenselektion, Vor-/Nachsorge}

Wie bei allen Licht- und Laser-basierten Technologien ist der Hauttyp des Patienten ein wichtiges Kriterium zur Risikoabwägung möglicher Nebenwirkungen. Gebräunte oder dunkelhäutigere Patienten (Fitzpatrick III-VI) sollten, wenn überhaupt, deutlich milder und zurückhaltender gelasert werden als hellhäutige Patienten [10]. Hinsichtlich des Risikos einer Pigmentverschiebung ist die Durchführung einer Probelaserung (intraindividuell) vor dem eigentlichen Lasereingriff zur Testung der Verträglichkeit immer ratsam. Hier empfiehlt sich auch, eine entsprechende Fotodokumentation vornehmen zu lassen. Ebenso entscheidend zur Vorbeugung möglicher post-inflammatorischer Hyper- und/oder Hypopigmentierungen ist eine ausreichende Sonnenkarenz von mindestens 4 Wochen vor und 12 Wochen nach der Behandlung. Bei Risikopatienten ist 
ggf. eine vorbereitende Therapie mit hydrochinoinhaltigen Externa zur Verminderung der Melanozyten-Stimulation nach Lasereingriff zur erwägen. Bei der Patientenaufklärung sollte auch nach immunologischen wie entzündlichen Komorbiditäten gefragt werden. Beim Vorliegen eines Lichen rubers oder einer Psoriasis vulgaris könnte der Lasereingriff dies im Sinne eines Köbner-Phänomens triggern. Zudem sollte nach der allgemeinen Wundheilung, Narbenheilung (cave: Keloide) und einer möglichen Sonnenallergie gefragt werden. Die Medikamentenanamnese umfasst blutverdünnende Medikamente, aber auch speziell phototoxische (z. B. Doxycyclin) oder photosensibilisierende (z.B. Hydrochlorothiazid, Furosemid) Medikamente und Homöopathika (Johanniskraut). Bei Aknepatienten sollte eine systemische Therapie mit Isotretinoin aufgrund seines phototoxischen Potenzials sowie seines ungünstigen Einflusses auf die Wundheilung mindestens 2 Monate zurückliegen. Ferner ist die Abfrage bekannter Allergien (Lokalanästhetika, Antibiotika) notwendig. Bei akuten Hautinfekten sollte generell von einem elektiven Lasereingriff abgesehen werden. Zur Vermeidung einer (Lippen-)Herpesinfektion sollte bei entsprechender Vorgeschichte eine „perioperative“ orale Prophylaxe mit Aciclovir $400 \mathrm{mg}(1-1-1)$ für insgesamt 5 Tage erfolgen. Bei großflächiger Laserbehandlung empfiehlt sich zum Schutz vor Hautinfektionen (bakterielle Superinfektion) eine orale Infektionsprophylaxe mittels Breitspektrum-Antibiotikum wie z.B. Cefuroxim $500 \mathrm{mg}$ (1-0-1), ebenfalls über 5 Tage. Wie schon erwähnt, handelt es sich bei der ablativen, fraktionierten $\mathrm{CO}_{2}$-Laser-Behandlung um einen Eingriff mit Operationscharakter!

Da dieser durch die Hitzeentwicklung durchaus schmerzhaft ist, wird ein Lokalanästhetikum in Cremeform (z.B. PliaglisCreme $^{\circledR}$, Galderma; cave: keine EMLA-Creme ${ }^{\circledR}$, da hierdurch keine ausreichende anästhetische Wirkung!) eine Stunde vor der Laserbehandlung aufgetragen, ggf. ergänzt durch eine orale Analgesie wie z. B. Paracetamol 1g, ebenfalls 30-60 Minuten vor dem Eingriff. Die Haut wird sorgfältig gereinigt und mit einem Antiseptikum desinfiziert. Während des Eingriffs ist es wichtig, die Haut zu kühlen (z. B. Zimmer-Kühlung, Firma Zimmer) und zugleich das bei der Gewebeabtragung anfallende Laser-Pyrolyse-Aerosol, das eine Mischung aus gas-, dampfund partikelförmigen Stoffen darstellt, mittels spezieller Rauchgasabsaugevorrichtung zu beseitigen. Zum Eigenschutz eignen sich zudem Atemschutzmasken. Die Übertragung von Virusinfektionen durch das Laseraerosol ist sowohl klinisch als auch experimentell eindeutig dokumentiert [1].

Je nach Indikation und Hauttextur erfolgen mehrere LaserDurchgänge nacheinander. Die verdampften Hautschichten werden mittels kochsalzgetränkter Tupfer vorsichtig entfernt. Um Demarkierungszonen zu vermeiden, werden die Randbereiche oft mit niedrigeren Energiedosen ausgestrichen. Die Dauer der Behandlung variiert je nach Umfang erheblich und beträgt in der Regel zwischen 20 und 60 Minuten.

Direkt nach der Behandlung wird eine antibiotische Hautcreme (z. B. Fucidine ${ }^{\circledR}$-Creme) zum lokalen Schutz vor Hautinfektionen aufgetragen. Je nach Lokalisation eignet sich auch das Anlegen eines Verbandes, mit Unterlage einer Wundgaze, um das Ankleben des Wundexsudats an den Verband und somit das Einreißen der Haut zu vermeiden. Eine zusätzliche Kühlung mittels Kühlbeuteln kann zusätzlich Linderung schaffen. Vor allem in den ersten 48 Stunden nach der Laserbehandlung nässt die Haut. Die seröse Flüssigkeit kann z. B. mit Essigwasser (1 Esslöffel Weißweinessig auf 1 Liter abgekochtes, lauwarmes Wasser) $3 \times$ täglich vorsichtig abgetupft werden, was auch der Bildung einer Pomadenkruste entgegenwirkt. Bis der Wundschorf abgefallen ist, empfiehlt es sich, die antibiotische Hautcreme $3 \times$ täglich anzuwenden. Der Schorf verbleibt in der Regel einige Tage bis eine Woche auf der behandelten Haut und sollte auf keinen Fall aktiv durch Kratzen oder Reiben entfernt werden, da sonst die Gefahr einer Narbenbildung besteht. Sobald dieser abgefallen ist, zeigt sich die Haut darunter für ca. 4-6 Wochen zartrosa. Diese bedarf wiederum einer speziellen, regenerierenden Pflege. Ein täglicher Sonnenschutz mit LSF 50+ ist ebenfalls für die nächsten 6 Monate nach Behandlung notwendig. Der Zustand der Haut ist vergleichbar mit einer Verbrennung zweiten Grades. Die Haut regeneriert sich binnen 1 - 2 Wochen. Fortbestehende störende Erytheme können mittels Lasern oder hochenergetischen Blitzlampen gebessert werden. Frühestens nach 3 Monaten lässt sich der finale Erfolg der Behandlung beurteilen. Ggf. sind auch mehrmalige Lasereingriffe zum Erhalt eines gewünschten kosmetischen Resultats notwendig und sollten im Vorfeld mit dem Patienten besprochen werden.

\section{Spezielle Indikationen}

\section{Ästhetik-Skinrejuvenation, insbesondere der Perioral-/Periorbitalregion; Botulinumtoxin Typ A als Adjuvans, Blepharoplastik}

In den letzten Jahren hat sich das Verständnis der komplexen Alterungsprozesse des menschlichen Gesichts mehr und mehr vertieft. Die meisten morphologischen und physiologischen Änderungen in der alternden Haut ergeben sich aus einer Kombination von intrinsischen und extrinsischen (z. B. UV-Bestrahlung, Nikotin) Faktoren [11]. Sie spielen sich auf zellulärer und nichtzellulärer Ebene ab, erfassen alle Schichten der Haut und sind sowohl mikro- als auch makroskopisch sichtbar. Die Epidermis wird dünner und die Dermis unterliegt massiven Änderungen im elastischen und kollagenen Fasergerüst, während die Subkutis zunehmend an Volumen verliert. Zur Verbesserung der Hautstruktur, von Dyschromien und zur allgemeinen Glättung bzw. Straffung der Gesichtshaut (Skin-Resurfacing) werden heutzutage aufgrund der überzeugenden Studienlage und der ausgesprochen guten Resultate zunehmend abtragende, fraktionierte $\mathrm{CO}_{2}$-Laser empfohlen $[12,13]$. Der durch die Hitzekoagulation erreichte „Shrinking“-Effekt sowie die verminderte Blutungsneigung machen die $\mathrm{CO}_{2}$-Laser besonders effektiv im Vergleich zu z.B. Er:YAG-Lasern. Durch die winzigen Verletzungspunkte der Fraktionierung werden die zerstörten Zellen abtransportiert und somit Dyschromien gemildert. Die Haut erneuert sich von innen heraus und wirkt jünger und frischer. Zudem ist die Ausfallzeit, insbesondere bei Behandlungen im schwer zu kaschierenden Gesichtsbereich, gegenüber dem klassischen Verfahren des ablativen Resurfacings deutlich verkürzt.

Ein besonderes Augenmerk wird auf die stark bewegten Areale des Gesichts - die Perioral- und Periorbitalregion - gelegt. Der Zug der mimischen Muskulatur stellt in diesen Arealen 

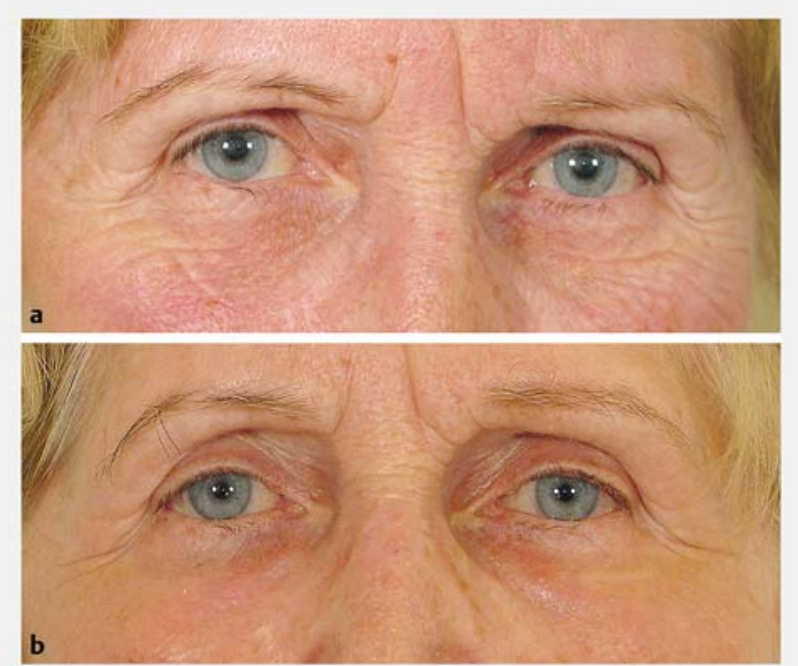

- Abb. 3 Fraktionierte $\mathrm{CO}_{2}$-Laserbehandlung der Periorbitalregion a vor Therapie. b Nach $\mathrm{CO}_{2}$-Lasertherapie (und Botulinumtoxin $\mathrm{A}$ als Adjuvans).

einen wichtigen pathologischen Faktor zur Entstehung der Falten - und deren Wiederkehr - dar. Aus diesem Grunde wird hier ein Kombinationsansatz aus Laserbehandlung und Botulinumtoxin Typ A angewendet. Wird die Haut zuvor durch Botulinumtoxin ruhiggestellt, kann die nachfolgende Heilung (Kollagen-Remodeling) effektiver erfolgen. Wird die Haut nicht ruhiggestellt, kommt es wegen des weiterhin bestehenden Muskelzuges zur erneuten Bildung von Falten. Eine kontinuierliche Erhaltungstherapie mit Botulinumtoxin im Abstand von 6-12 Monaten führt zudem zu einem besseren Langzeitergebnis $[14,15]$ ( $>$ Abb. 3 ).

Die Blepharoplastik (Augenlidstraffung) ist eine der am häufigsten nachgefragten plastischen Eingriffe in der Altersgruppe zwischen dem 40. und 60. Lebensjahr und wird von Ärzten unterschiedlicher Fachrichtungen ausgeführt. Das Ziel ist es, das jugendliche, frische Aussehen des Augenbereichs unter Beachtung eines funktionell einwandfreien und natürlich aussehenden Ergebnisses wiederherzustellen. Die Stellung des gepulsten $\mathrm{CO}_{2}$-Lasers als Laserskalpell wurde 1983 von Baker erstmals beschrieben und ein Jahr später im „Yearbook of Ophthalmology“ veröffentlicht. Die Behandlung kann sowohl im Bereich der Oberlider (z. B. bei Schlupflidern) als auch im Bereich der Unterlider (z. B. bei Tränensäcken) durchgeführt werden. Der ultragepulste $\mathrm{CO}_{2}$-Laserstrahl wird hierfür als Schneidewerkzeug fokussiert verwendet. Er bietet eine ausgezeichnete chirurgische Präzision. Durch die ultrakurz gepulste Technologie können die Nebenwirkungen durch Hitzekoagulation auf das umliegende Gewebe minimiert werden. Wird er defokussiert verwendet, können zusätzlich sich vorwölbende Fettdepots durch Verdampfung reduziert werden. Ein weiterer wesentlicher Vorteil durch die Verwendung eines Laserskalpells liegt in der Reduzierung intraoperativer Blutungen und dadurch der verbesserten intraoperativen Darstellung und Identifizierung der Lidanatomie sowie der im Heilungsverlauf verringerten post-operativen Schwellungen und Hämatome. Das Risiko an Nachblutungen wird ebenfalls verringert. Die Laser-assistierte Blepharoplastik (LABP) erfolgt unter Lokalanästhesie mit i.v. Sedierung oder in Allgemeinnarkose. Der Augapfel muss während der Lasereingriffs durch eine Metallschale geschützt werden, um eine Verletzung der Hornhaut zu vermeiden und das Risiko einer Chemosis zu verringern. Ein zusätzlicher Vorteil der LABP gegenüber der klassisch chirurgischen Unterlidplastik - bei der zwar überschüssige Haut entfernt werden kann, aber die Qualität der verbleibenden periorbitalen Haut nicht verbessert werden kann - ist, dass zudem ein ergänzendes Lid- oder periorbitales Skin-Resurfacing kombiniert werden kann, um somit optimale kosmetische Resultate zu erzielen [16].

\section{Narbenbehandlung - atrophe Narben, linear und flächig hypertrophe Narben}

Narben sind das verbleibende Zeichen tieferer Verletzungen der Haut und entwickeln sich im Rahmen der normalen Wundheilung als deren Endzustand. Das komplex verflochtene kollagene Netzwerk der Haut wird durch ein minderwertiges, faserreiches Ersatzgewebe (Fibrose) ersetzt. Neben frischen unreifen Narben, die im Laufe der Heilung in reife Narben übergehen und in der Regel keiner weiteren Behandlung bedürfen, kann es in Abhängigkeit von Lokalisation, Ursache, Heilungsverlauf und individueller Anlagen jedoch zu einer gestörten Wundheilung mit unzureichender oder auch übermäßiger Bildung von Narbengewebe kommen. Die Behandlung dieser atrophen und hypertrophen (linear oder flächig) Narben stellt trotz langjähriger Erfahrung nach wie vor eine therapeutische Herausforderung für den behandelnden Arzt dar. Ziel der Narbenbehandlung ist es, Größe, Ausdehnung, Volumen oder Substanzdefekte zu reduzieren und Beschwerden wie Juckreiz, Schmerzen und Spannungsgefühl zu verringern. Funktionelle (z.B. Kontrakturen) und ästhetische (Stigmatisierung) Einschränkungen können ebenfalls Grundlage für den Behandlungswunsch sein.

Der Entstehung von atrophen (eingesunkenen) Narben liegt häufig eine länger bestehende Entzündungsreaktion (schwere Akne oder Varizelleninfektion) in tieferen Schichten der Haut zugrunde. Sie treten, entsprechend ihrer Grunderkrankung, vor allem im Gesicht und Rückenbereich auf. Die anhaltende intrakutane Entzündungsreaktion führt zu einer Zerstörung von tieferen Strukturen der Haut. Es kommt zu einer verminderten Matrixregeneration und lokal verminderten Kollagenproduktion und somit zur Destruktion der dermalen Architektur. Zudem atrophiert subkutanes Fett im Zuge der Entzündung. Durch die zusätzliche Kontraktur des Narbengewebes entstehen so die kleinen Weichteildefekte, die an der Hautoberfläche als atrophe, eingesunkene Narben imponieren [17]. Atrophe Aknenarben treten häufig auf und sind zum Teil sehr stigmatisierend für die betroffenen Patienten. Sie werden in 3 Typen (wurmstichartig, varioliform, wellenartig) unterteilt. Ausgehend vom Narbentyp werden die geeigneten Therapieoptionen gewählt. Operative Verfahren wie die Stanzexzision eignen sich für einzelne, tief eingesunkene, wurmstichartige Narben (auch „ice-pick scars“ genannt). Die Subzision (Unterminieren von Verwachsungen unterhalb der eingesunkenen Narbe mittels einer speziellen Nadel) kommt bei tieferen, wel- 
lenartigen Narben zum Einsatz, die Dermabrasion bei großflächigen, tiefen Akne-Narben. Zum Goldstandard in der Therapie atropher (varioliformer oder schüsselförmiger) Akne-Narben zählt inzwischen die ablative, fraktionierte $\mathrm{CO}_{2}$-Lasertherapie [18-20]. Durch den thermischen Effekt des fraktionierten $\mathrm{CO}_{2}$-Lasers kommt es zu einer Stimulation des Kollagenumbaus und einer Hautstraffung und somit zur Schrumpfung der eingesunkenen Narbe und einer Nivellierung des Hautreliefs [19]. Seine ablative Komponente dient zusätzlich zur Glättung der Hautoberfläche. Bei unterschiedlichen Narbenentitäten werden oftmals auch die einzelnen therapeutischen Maßnahmen kombiniert. Zum Beispiel können einzelne ice-pick scars vor der geplanten $\mathrm{CO}_{2}$-Laserbehandlung mittels Stanzexzision entfernt werden, was das Endresultat durchaus deutlich verbessern kann. Da bei den fraktionierten $\mathrm{CO}_{2}$-Lasern die Umbauprozesse des Narbengewebes im Vordergrund stehen, benötigt es 3-6 Monate, bis die finalen Ergebnisse der Behandlung ersichtlich sind. Je nach Schweregrad des Ausgangsbefundes sind 2-4 Lasersitzungen notwendig, um den gewünschten ästhetischen Effekt zu erzielen. Da Akne-Narben oft nicht das gesamte Gesicht einnehmen, sondern gerne auf die Wangen- oder Schläfenregion begrenzt sind, ist das „Ausblenden“ im Randbereich der jeweils zu behandelnden Areale besonders wichtig, um keine länger anhaltenden, ästhetisch unschönen Demarkierungslinien zu generieren. Chemische Peelings sowie das klassische Medical Needling mittels Nadelroller (mind. 1,5 mm Nadellänge) stellen hier ergänzend erwähnt auch eine geeignete Therapieoption für milde, atrophe Akne-Narben dar. Der Vorteil des Medical Needlings gegenüber dem Laserverfahren liegt in der geringeren Downtime und einem geringeren Risikopotenzial. Nachteilig ist, dass in jedem Fall mehrere Behandlungen erfolgen müssen (ca. 4-6) und auch oftmals nur bei milderen, flacheren „Akne-Närbchen“ zufriedenstellende Ergebnisse zu erzielen sind. Für alle Entitäten bleibt jedoch die Prävention ungleich effizienter als die jeweilige Behandlung. Durch die frühzeitige, leitliniengerechte Behandlung der unterschiedlichen Stadien der Akne lassen sich atrophe Narben heute häufig vermeiden oder zumindest signifikant verringern ( $\triangleright$ Abb.4).

Eine hypertrophe Narbe wird als Bindegewebswucherung definiert, die die Grenze der ursprünglichen chirurgischen oder Verletzungswunde nicht überschreitet. Lineare hypertrophe Narben zeigen eine strangartige Wulstbildung im Rahmen der Grenzen des ursprünglichen Traumas. Sie sind häufig gerötet und assoziiert mit Juckreiz und Schmerzen. Nachdem sie innerhalb weniger Wochen stark an Größe zugenommen haben, zeigen sie nach einer statischen Phase häufig einen Größenregress. Flächige hypertrophe Narben entstehen infolge von flächigen Traumata, beispielsweise nach Verbrennungen oder Verätzungen. Sie zeigen eine unregelmäßige, häufig stark gerötete Oberfläche mit zum Teil deutlicher Verhärtung und Strangbildung. Keloide werden häufig mit hypertrophen Narben verwechselt. Im Unterschied zu hypertrophen Narben überschreiten sie allerdings typischerweise die Grenze des ursprünglichen Traumas und wachsen lippenförmig, häufig über mehrere Jahre, immer weiter. Sie sind derb, wulstig, stark gerötet und verursachen häufig Juckreiz und Druckschmerzen. Zur Behandlung hypertropher Narben steht eine Vielzahl von

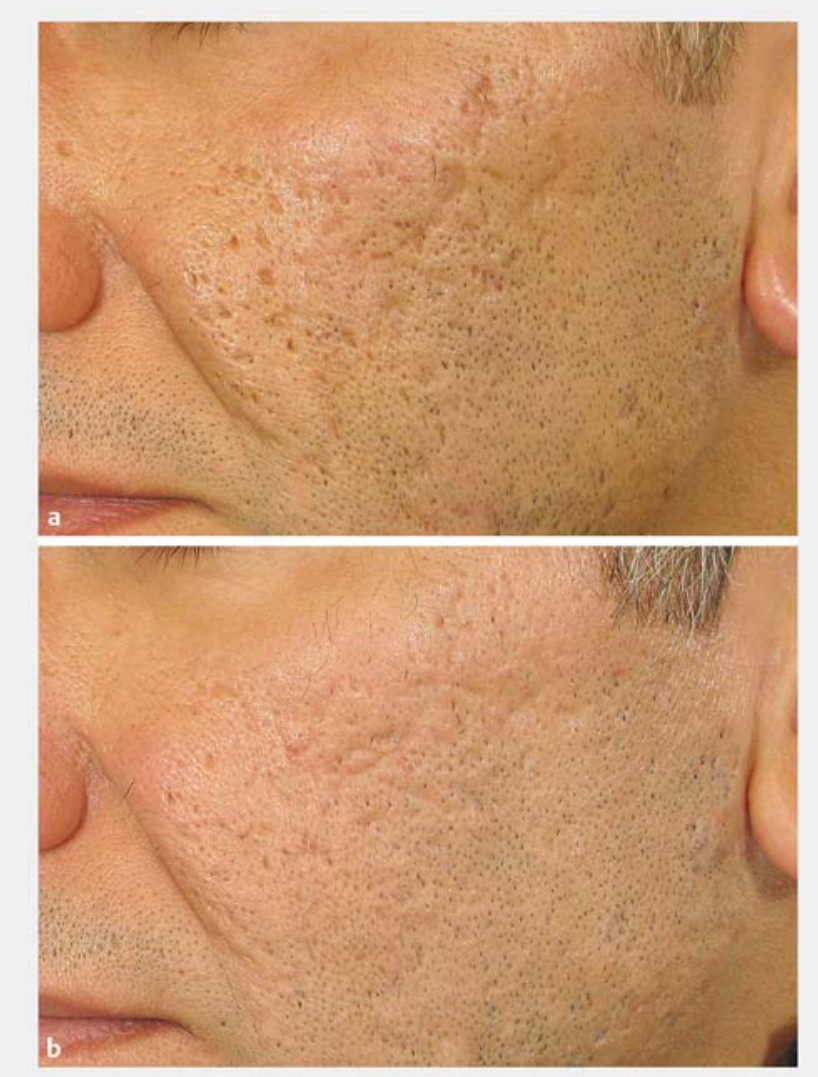

- Abb. 4 Fraktionierte $\mathrm{CO}_{2}$-Laserbehandlung bei Akne-Narben: a vor Therapie. b Nach Punch-Biopsie („,ice-pick scars“) und $2 \times \mathrm{CO}_{2}$-Laserbehandlung.

Behandlungsmöglichkeiten zur Verfügung [17]. Silikonhaltige Externa stellen die Erstlinientherapie dar. Die Verwendung von Zwiebelextrakt-haltigen Externa kann für die Behandlung aktiver hypertropher Narben und zur Prophylaxe empfohlen werden. Bei flächigen hypertrophen Narben zeigte die Drucktherapie gute Ergebnisse zur Narbenprophylaxe. Sie erfordert jedoch speziell angepasste Kleidung, die nach Möglichkeit bis zu 24 Stunden täglich getragen werden sollte und dies über einen langen Zeitraum, was der Compliance des Patienten bedarf. Die intraläsionale Injektion kristalliner Glukokortikosteroide in Kombination mit Kryotherapie stellt seit den 60er-Jahren eine etablierte Behandlungsmethode zur Zweitlinientherapie von hypertrophen Narben dar. Intraläsionale Injektion von 5-Fluorouracil (5-FU) wird seit 1989 eingesetzt [17, 21, 22].

Basierend auf zahlreichen Studien und den aktualisierten internationalen Leitlinien werden neben Farbstoff- und Nd:YAGLasern erstmals fraktionierte Laser zur Zweitlinientherapie für inaktive, lineare und flächige hypertrophe Narben empfohlen $[17,23,24]$. Ein Behandlungsansatz, der schon seit längerer Zeit zur Behandlung von atrophen (Akne-) Narben erfolgreich eingesetzt wird. Wie auch bei deren Behandlung scheint der fraktionierte $\mathrm{CO}_{2}$-Laser dem Er:YAG-Laser aufgrund der gezielten Wärmeentwicklung und der größeren Eindringtiefe (z.B. UltraPulse ${ }^{\circledR}$, Scaar FX, Lumenis, Eindringtiefe $4 \mathrm{~mm}$ ) überlegen 
zu sein [24]. Klinisch lassen sich neben einem homogeneren Narbenbild funktionelle Verbesserungen, z. B. im Bereich von Kontrakturen, erreichen. Verantwortlich dafür scheinen die relativ komplexen Veränderungen im Narbengewebe nach der Laserbehandlung zu sein. Laut aktueller Studien bewirken sie eine Veränderung verschiedener Zytokin- und Wachstumsfaktorkonzentrationen, eine Wiederherstellung der ursprünglichen (physiologischen) Kollagenarchitektur und eine Zunahme von elastischen Fasern [24]. Ihr Einsatz in der Behandlung von (aktiven) Keloiden sollte aber weiterhin mit größter Zurückhaltung erfolgen. Wegen der ausgeprägten Rezidivneigung sind hierdurch keine langfristigen Therapierfolge zu erwarten. In Erwägung gezogen werden kann jedoch eine Laserbehandlung zum Debulking größerer Läsionen, sofern parallel weiter präventive Maßnahmen wie intraläsionale Injektion von Triamcinolonacetonid (Erstlinientherapie bei Keloiden) angewandt werden [17].

Insbesondere bei den bisher schwer zu therapierenden Verbrennungsnarben stellt die ablative, fraktionierte $\mathrm{CO}_{2}$-Lasertherapie eine vielversprechende Therapieoption dar.

Brandwunden sind leider eine weltweite, sehr häufige Verletzung [25]. Bis zu 77\% der Brandverletzten entwickeln pathologische (Brand-)Narben [26]. An exponierten Hautarealen sind sie zudem äußerst stigmatisierend für die Betroffenen. Brandnarben sind meist relativ großflächig und können im Bereich von Gelenken zu Bewegungseinschränkungen durch sogenannte Narbenkontrakturen führen. Insofern sind Vereisung und Injektion von Kortikosteroiden nur in umgrenzten Arealen sinnvoll. Auch wenn bei großflächiger Zerstörung der Hautarchitektur durch Verbrennung sicherlich mehrere Laserbehandlungen (in ca. 3-monatigen Abständen) notwendig sind, um klinisch überzeugende Ergebnisse zu erlangen, sind diese sehr gut unter ambulanten Bedingungen durchführbar, wie wir auch in unserer Arbeitsgruppe feststellten [27].

\section{Sonstige Anwendungsbereiche: Abtragung von benignen und prämalignen Hautveränderungen}

Aufgrund der schon beschriebenen Vorteile der zielgerichteten Gewebeabtragung in Kombination mit Blutstillung und minimaler Kollateralschädigung des umliegenden Gewebes, werden moderne, ablative $\mathrm{CO}_{2}$-Resurfacing Laser auch effektiv bei der Re-Konturierung eines Rhinophyms angewendet [15]. Zwar gelten die tangentiale Abtragung (Shave Exzision), Kürettage oder auch Kryotherapie als „First line“-Therapien in der Behandlung von epidermalen Naevi, seborrhoischen Keratosen, benignen Talgdrüsenhyperplasien, Syringomen und Xanthelasmen, aber besonders in ästhetisch delikaten Bereichen wie den Augenlidern, der Nase und den Lippen bieten die ablativen $\mathrm{CO}_{2}$-Laser die schon beschriebenen Vorteile - zielgerichtete Abtragung und Blutstillung. Tuberöse Angiome stellen auch eine Therapieindikation dar, wobei auf die neue Therapiemöglichkeit mittels topischen Rapamycins bei mit tuberöser Sklerose assoziierten Angiofibromen hingewiesen werden muss. Präkanzerosen wie die Cheilitis actinica, die aktinischen Keratosen und der Morbus Bowen stellen eine weitere klinische Anwendung für diese Laserverfahren dar, insbesondere seit deren kombinierter Anwendung mit der klassischen photodynamischen Therapie (PDT).

\section{"Laser-assisted drug delivery“ - neues Konzept der Dermatopharmakotherapie}

Die Behandlung von Hauterkrankungen durch topisch applizierte Externa ist eine der wichtigsten Therapieoptionen in der Dermatologie. Aufgrund der oftmals geringen Bioverfügbarkeit von Lokaltherapeutika wurden zahlreiche Methoden entwickelt, um die Penetration dieser Substanzen zu verbessen von Folien- (okklusiv) Technik bis hin zum Microneedling. Fraktionierte Laser stellen ein neuartiges, elegantes Verfahren dar, um die dermale Barriere in kontrollierter Weise zu überwinden, um somit die Eindringtiefe von lokal applizierten Pharmaka als auch deren dermale Bioverfügbarkeit im Sinn eines „Laser assisted drug delivery“ (LADD) zu steigern. Diese Technik kommt heutzutage schon verstärkt bei der photodynamischen Therapie (PDT) zur Behandlung von Hautkrebsvorstufen zum Einsatz. Um die Wirkung der konventionellen PDT mittels fraktionierter $\mathrm{CO}_{2}$-Laser zu verstärken und zu verbessern (LADD-PDT), wird die Haut vor dem Auftragen des Photosensibilisators mittels Laser behandelt. Durch die somit erzeugten mikroskopisch kleinen Kanäle in der Haut (MTZ) kann die Creme (der Wirkstoff) tiefer und effektiver in das Gewebe eindringen und das Ergebnis optimieren [28]. Die Vorteile des LADD werden auch für viele andere Substanzen wie Lidocain, 5-FU, Triamcinolon, Diclofenac, Opiate, MTX, Vitamin C, Imiquimod, und natürlich auch für Cosmeceuticals ausgiebig untersucht [29]. Es sind jedoch noch weitere, genauere Untersuchungen nötig, um für die jeweiligen Substanzen, u.a. abhängig von deren Molekulargewicht, die geeigneten Parameter (z. B. density \%) im Laser-Setting zu bestimmen, um deren optimale Eindringtiefe (intrakutan, transkutan) und somit Wirkort und gewünschte WirkstoffKonzentration zu bestimmen [30].

\section{Fazit}

Durch stetige technische Weiterentwicklung hat sich der bereits seit den $1960 \mathrm{er}$-Jahren verbreitete $\mathrm{CO}_{2}$-Laser als Standardwerkzeug zur Behandlung unterschiedlicher dermatologischer Indikationen etabliert. Sein Einsatz im fraktionierten Modus kombiniert eine hohe Effektivität der Therapie mit einer niedrigen Rekonvaleszenzzeit. Die Anwendung ist zudem schmerzarm und sehr gut ambulant durchführbar. Zum Einsatz kommt die fraktionierte $\mathrm{CO}_{2}$-Lasertherapie mittlerweile für eine Vielzahl von Indikationen wie zum Beispiel unterschiedliche gutartige und prämaligne Hautveränderungen, Narben sowie die straffende Behandlung der alternden Haut durch das LaserSkin-Resurfacing. Im Rahmen dieser Behandlungen spielt nicht nur die abtragende Vaporisierungswirkung, mit der Möglichkeit Blutgefäße zu koagulieren, eine Rolle, sondern auch die komplexen Stimulationsvorgänge im Bereich der tiefen Dermis, die eine reorganisierende Wirkung auf das veränderte Hautgerüst haben. Durch langjährige Erfahrung und immer neue technische Innovationen, die zu einer zunehmenden Reduktion der Nebenwirkungen und einer Verbesserung der Behandlungseffektivität geführt haben, ist die Therapie mit dem fraktionierten $\mathrm{CO}_{2}$-Laser daher aus der modernen Dermatologie nicht mehr wegzudenken. 
Interessenkonflikt

G. G. Gauglitz: Berater und Vortragender für Merz Pharmaceuticals, Sinclair Pharma, Galderma, Lumenis, Candela, Asclepion, Cynosure, Pollogen, Johnson \& Johnson, Almiral, Biofrontera, Urgo GmbH and MediWound.

S. Steckmeier und J. Poetschke geben an, dass kein Interessenkonflikt besteht.

Literatur

[1] Landthaler M. Laser und photodynamische Therapie. In: Plewig G et al., Hrsg. Braun-Falco's Dermatologie, Venerologie und Allergologie. 6. Auflage Heidelberg: Springer; 2012: 1928-1932

[2] Stewart N, Lim AC, Lowe MP et al. Laser and laser-like devices: Part one. Australian Journal of Dermatology 2013; 54: 173 - 183

[3] Fitzpatrick RE, Golmann MP, Satur NM et al. Pulsed carbon dioxide laser resurfacing of photoaged facial skin. Arch Dermatol 1996; 132 $395-402$

[4] Manstein D, Herron GS, Sink RK et al. Fractional Photothermolysis: A new concept for cutaneous Remodeling using microscopic patterns of thermal injury. Lasers Surg Med 2004; 34: 426-438

[5] Hohenleutner U. Innovationen in der dermatologischen Lasertherapie. Hautarzt 2010; 5: 410-415

[6] Laubach H], Tannous Z, Anderson RR et al. Skin responses to fractional photothermolysis. Lasers Surg Med 2006; 38: 142 - 149

[7] Helbig D, Paasch U. Molecular changes during skin aging and wound healing after fractional ablative photothermolysis. Skin research and technology: official journal of International Society for Bioengeneering and the Skin (ISBS) and International Society for Digital Imaging of Skin (ISDIS) and International Society for Skin Imaging (ISSI) 2011; 17: $119-128$

[8] Ozog DM, Lin A, Chaffins ML et al. Evaluation of clinical results, histological architecture, and collagen expression following treatment of mature burn scars with a fractional carbon dioxide Laser. JAMA Dermatol 2013; 149: 50 - 57

[9] Paasch U. Fraktionierte nicht ablative Laser. ästhet dermatol kosmetol 2015; 3: $36-45$

[10] Kautz G, Kautz I. Intense Pulsed Light. ästhet dermatol kosmetol 2015; 7: 17-18

[11] Gauglitz GG. Panfacial aesthetic minimal-invasive approaches. MMW Fortschr Med 2015; 157: $44-47$

[12] Hunzecker CM, Weiss ET, Geronemus RG. Fractionated $\mathrm{CO}_{2}$-laser resurfacing : our experiance with more than 2000 treatments. Aesthetic Surg J 2009; 29: $317-322$

[13] Clementoni MT, Lavagno R, Munavalli G. A new multi-modal fractional ablative $\mathrm{CO}_{2}$-laser for wrinkle reduction and skin resurfacing. J Cosmet Laser Ther 2012; 14: 244 - 252

[14] Sommer B, Bergfeld D, Sattler G. 5 Botulinumtoxin Typ A als Adjuvans, 5.5 bei Rejuvenierungsverfahren der Dermis. Botulinumtoxin in der ästhetischen Medizin. Stuttgart: Thieme; 2006: 74-75
[15] Sebaratnam DF, Lim AC, Lowe PM et al. Lasers and laser-like devices: Part two. Australian Journal of Dermatology 2014; 55: 1-14

[16] Attrasch C, Fratila AM. Indikationen und Vorteile der $\mathrm{CO}_{2}$-Laserassistierten Unterlidblepharoplastik in Kombination mit UltraPulse Encore $^{\mathrm{TM}} \mathrm{CO}_{2}$-Laser Skin Resurfacing der periorbitalen Haut. Face 2011; 3: $32-35$

[17] Poetschke J, Gauglitz GG. Aktuelle Optionen zur Behandlung pathologischer Narben. Journal of the German Society of Dermatology 2016; 14: $467-478$

[18] Lee HS, Lee JH, Ahn GY et al. Fractional photothermolysis for the treatment of acne scars: A report of 27 Korean patients. Journal of Dermatological Treatment 2008; 19: $45-49$

[19] Reinholz M, Schwaiger H, Heppt MV et al. Comparison of two kinds of lasers in the treatment of acne scars. Facial Plast Surg 2015; 31805: $523-531$

[20] Manuskiatti W, Triwongwaranat D, Varothai S et al. Efficacy and safety of a carbon-dioxide ablative fractional resurfacing device for the treatment of atrophic acne scars in Asians. J Am Acad Dermatol 2010; 63: $274-283$

[21] Gold MH, Berman B, Clementoni MT et al. Updated international clinical recommendations on scar management: part 1 - evaluating the evidence. Dermatologic surgery: official publication for American Society for Dermatologic Surgery (et al.) 2014; 40: 817-824

[22] Gold MH, Mc Guire M, Mustoe TA et al. Updated international clinical recommendations on scar management: part 2 - algorithms for scar prevention and treatment. Dermatologic surgery: official publication for American Society for Dermatologic Surgery (et al.) 2014; 40: $825-831$

[23] Anderson RR, Donelan MB, Hivnor C et al. Lasertreatment of traumatic scars with an emphasis on ablative fractional laser resurfacing: consensus report. JAMA Dermatol 2014; 150: 187-193

[24] Gauglitz GG. Management of keloids and hypertrophic scars: current and emerging options. Clin Cosmet Investig Dermatol 2013; 6: 103 114

[25] American Burn Association. Burn incidence and treatment in the United States 2016. http://www.americanburn.org

[26] Gangemi E, Gregori D, Berchialla P et al. Epidemiology and risk factors for pathologic scaring after burn wounds. Archives of Facial Plastic Surgery 2008; 10: $93-102$

[27] Poetschke J, Dornseifer U, Clementoni MT et al. Ultrapulsed fractional ablative carbon dioxide laser treatment of hypertrophic burn scars: evaluation of an in-patient controlled, standardized treatment approach. Lasers in Medical Science. London: Springer; 2017. doi:10.1007/s 10103-017-2204-7

[28] Haedersdal M, Sakamoto FH, Farinelli WA et al. Fractional CO2 LaserAssisted Drug Delivery. Lasers in Surgery and Medicine 2010; 42: $113-122$

[29] Sklar LR, Burnett CT, Waibel JS et al. Laser assisted Drug Delivery: A Review of an evolving technology. Lasers in Surgery and Medicine 2014; 46: 249-262

[30] Haak CS, Bhayana B, Farinelli WA et al. The impact of treatment density and molecular weight for fractional laser-assisted drug delivery. Journal of controlled release 2012; 163: 335 - 341 